Rounson, G. N. \& LumB, M. (1953). J. gen. Microbiol. 9, 385-393.

\title{
The Oxidation of Lard Oil by Penicillium chrysogenum
}

\author{
By G. N. ROLINSON AND M. LUMB \\ Research Department, Bacteriology Division, Boots Pure \\ Drug Co. Ltd., Nottingham
}

SUMMARY: Under the conditions prevailing during the early stages of certain highly aerated penicillin fermentations, lard oil and oleic acid appear to be oxidized only slowly, or not at all, by suspensions of washed mycelium of Penicillium chrysogenum. Lard oil and oleic acid are both oxidized rapidly, however, under the conditions present at $48 \mathrm{hr}$. and after. The optimum $\mathrm{pH}$ for oxidation of lard oil by suspensions of washed mycelium c. $48 \mathrm{hr}$. old is $8 \cdot 1$ and for oleic acid, c. 6.4, but oxidation of both lard oil and oleic acid was apparent at the $\mathrm{pH}$ prevailing during the early stages of the fermentation $(5 \cdot 5-6 \cdot 0)$.

Mycelium from shaken flask fermentations (conditions of relatively low aeration) appeared to oxidize lard oil only slowly even during the later stages of the fermentation although oleic acid, which is the principal product of hydrolysis of lard oil, was oxidized rapidly.

The results suggest that enzymes involved in the oxidation of lard oil and oleic acid may be present only in relatively low concentration in young mycelium and be synthesized as growth takes place. The ability to oxidize lard oil also appears to be favoured by conditions of high aeration during growth.

It has been shown (Rolinson \& Lumb, 1953) that in highly aerated sporeinoculated penicillin fermentations, lard oil, added as a vehicle for the antifoam agent, is utilized as a major source of carbon by Penicillium chrysogenum. Suspensions of washed mycelium from similar highly aerated fermentations were also capable of oxidizing lard oil rapidly when it was added as the sole substrate, but washed mycelium grown under conditions of relatively low aeration (in shaken flasks) failed to show any increase in respiration over the endogenous rate when incubated with lard oil.

The present paper is concerned with further investigations upon factors affecting this oxidation of lard oil by $\boldsymbol{P}$. chrysogenum.

\section{METHODS}

Fermentation methods. Two types of fermentation equipment were used which provided widely different conditions of aeration. Conditions of high aeration were obtained in laboratory fermenters of the type described by Lumb \& Fawcett (1951) containing 3 l. medium with stirring at 1000 r.p.m. and an air flow of $6 \mathrm{l} . / \mathrm{min}$. Relatively low aeration was obtained by using $75 \mathrm{ml}$. medium in $250 \mathrm{ml}$. conical Pyrex or Hysil flasks on a rotary shaker having a speed of 220 r.p.m. and a throw of $1 \frac{1}{2}$ in. All fermentations were carried out at 24-26 $6^{\circ}$. The medium used in all the experiments contained : corn steep liquor (solids basis), $1.5 \%$; lactose, $3.0 \%$; glucose, $0.5 \%$; $\mathrm{NaNO}_{3}, \mathbf{0 . 3} \%$; $\mathrm{KH}_{2} \mathrm{PO}_{4}, 0.05 \% ; \mathrm{Na}_{2} \mathrm{SO}_{4}, 0.1 \% ; \mathrm{pH}$ adjusted to 5.8 . Phenylacetic acid was added to the stirred and aerated fermentations in additions of $0.05 \%$ every 
$12 \mathrm{hr}$. starting at the 24th hour. In the shaken flask fermentations, a concentration of $0.25 \%$ phenylacetic acid was included in the medium initially. The antifoam agent used was $2.5 \%$ cetyl alcohol in lard oil, added as required. All the fermentations were inoculated with spore suspensions prepared from freeze-dried master cultures of $P$. chrysogenum W 48-701.

Manometric methods. Conventional Warburg techniques were used as described by Umbreit, Burris \& Stauffer (1945). Suspensions of washed mycelium were prepared by centrifuging a sample of fermentation liquor and washing 5 times with $\mathrm{KH}_{2} \mathrm{PO}_{4}+\mathrm{NaOH}$ buffer or with a solution containing only the inorganic constituents of the medium (hereafter called salts solution). All measurements of oxygen uptake were made with manometers maintained at $26^{\circ}$, rocked at 120 strokes/min. This rate was found adequate in previous experiments to ensure that the rate of solution of oxygen in the liquid in the manometer flasks was not a limiting factor.

\section{RESULTS}

Experiments were carried out to determine the time taken for oxidation of lard oil to be apparent when it was added to washed mycelium. The mycelium used was $50 \mathrm{hr}$. old, taken from a highly aerated fermentation. After washing and suspending in $0.025 \mathrm{M}$-phosphate buffer $\mathrm{pH} 7.5$ the mycelium was incubated in Warburg manometers. A period of $20 \mathrm{~min}$. was allowed for equilibration of temperature. Endogenous respiration was measured for a further

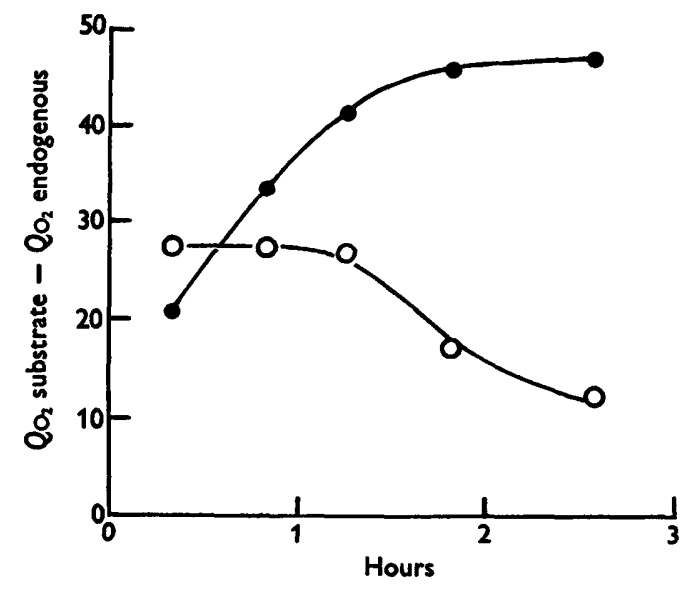

Fig. 1. Oxidation of lard oil over a prolonged period of time by washed mycelium of P. chrysogenum. $1 \%(\mathrm{v} / \mathrm{v})$, lard oil; - $-0.1 \%(\mathrm{v} / \mathrm{v})$, lard oil, $\mathrm{O}-\mathrm{O}$. (Endogenous $Q_{\mathrm{O}_{\mathbf{2}}}=$ 13.1.)

25 min., then the substrate was tipped in and readings were continued up to $50 \mathrm{~min}$. The oxygen uptake increased c. $150 \%$ almost immediately after the lard oil was added. Oxidation of lard oil over a longer period of time is shown in Fig. 1. Mycelium $46 \mathrm{hr}$. old from a highly aerated fermentation was washed and suspended in $0.025 \mathrm{M}$-phosphate buffer $\mathrm{pH} \mathrm{7.5}$ as in the previous experiment. The average endogenous $Q_{\mathrm{o}_{2}}$ of the mycelium, which remained almost 
The absence of any increase in oxygen uptake of young mycelium when lard oil was added as substrate may not indicate the complete absence of oxidation of this substrate. The endogenous respiration in these experiments was relatively high (typical of fungi). A substrate added to a suspension of washed mycelium might well be oxidized, but at the expense of substances otherwise contributing to the endogenous respiration, and thus result in no increase in overall rate of respiration. The apparent absence of lard-oil oxidation by young mycelium may only indicate a relatively lower rate of oxidation than occurs with the older mycelium.

The change in ability to oxidize lard oil and oleic acid may be due to the synthesis of the necessary enzymes during growth. Variations in enzyme activity with age have been reported for a number of bacterial enzymes (Gale, 1943) which show little or no activity in young cells but are formed during growth and reach a maximum at the time of cessation of cell division. In the experiments described here with $\boldsymbol{P}$. chrysogenum the period when the ability to oxidize lard oil increased also corresponded with the period of maximum rate of growth. However, such a variation in enzyme activity might not be due to variation in enzyme content but to changes in the conditions for enzyme action such as changes in $\mathrm{pH}$ value of the medium or permeability of the cells during the fermentation. During the initial $24 \mathrm{hr}$. the $\mathrm{pH}$ value of the fermentation is not optimum for oxidation of either lard oil or oleic acid, but the results shown in Fig. 4 suggest that the $\mathrm{pH}$ alone would not preclude enzyme activity being apparent at this stage of the fermentation, particularly with oleic acid as substrate. However, experiments with washed mycelium suspended in buffers do not rule out the possibility that the internal $\mathrm{pH}$ of the mycelium is not the same as that of the surrounding medium. The presence during the early stages of growth of chemical factors which might inhibit enzyme action also cannot be overlooked, although this might be expected to be minimized by the washing procedure.

Variation in permeability of the mycelium to lard oil and oleic acid might also be responsible for variations in enzyme activity with age. However, experiments showed that no appreciable period of time after adding substrate (lard oil) was required before an increased rate of oxygen uptake was observed with $50 \mathrm{hr}$. mycelium, yet with young mycelium no increase in respiration over the endogenous rate was found over $30 \mathrm{~min}$. after lard oil was added. It is also not unlikely that the whole fat does not penetrate the cell but is hydrolysed outside the cell membrane. Furthermore, if the variations in enzyme activity with age were due to changes in permeability it would also be necessary to postulate a decrease in permeability after $c .48 \mathrm{hr}$. when enzyme activity declined (Fig. 3).

The inability of young mycelium to oxidize lard oil is not due only to an inability to oxidize the constituent fatty acids at this stage. The fat is apparently not hydrolysed, because glycerol, which would be liberated by hydrolysis, was readily oxidized by young mycelium at pH 5.3 (Table 1).

Oxidation of oleic acid by mycelium grown under conditions of relatively low aeration showed a similar variation with age as seen with the mycelium 
time of sampling. The salts solution was used since it resembled the fermentation medium more closely than did the phosphate buffer. Each Warburg flask contained $1.0 \mathrm{ml}$. of the mycelial suspension and $1.0 \mathrm{ml}$. of salts solution. Oxygen uptake in the presence of lard oil and also glucose and oleic acid is shown in Fig. 3. Respiration in the absence of added substrate (endogenous respiration) was highest when the mycelium was young and declined slowly as the fermentation proceeded. With glucose as substrate (which increased oxygen uptake c. $85 \%$ over the endogenous rate) the maximum rate of oxidation also occurred with the young mycelium. With lard oil or oleic acid as substrate, however, the oxygen uptake of young mycelium showed no increase over the endogenous rate, and respiration was in fact slightly lower

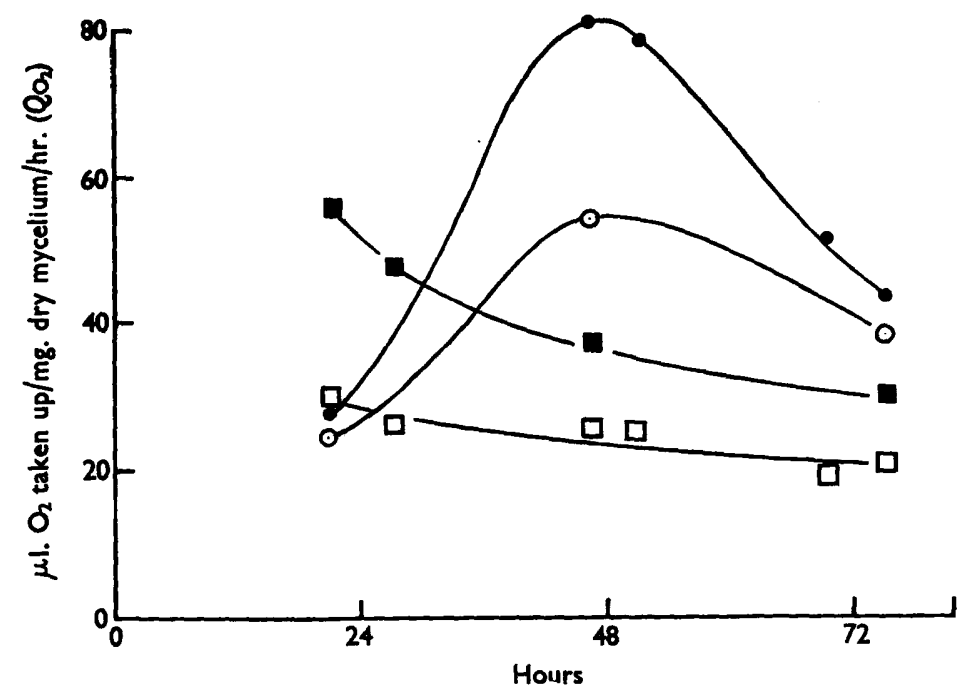

Fig. 3. Enzyme activities of washed mycelium of $P$. chrysogenum at different stages of a highly aerated fermentation. Endogenous respiration, $\square-\square$. Substrates: glucose, $1 \%(w / v), \square$; oleic acid, $1 \%(v / v)$, - ; lard oil, $1 \%(v / v), 0-0$.

in the presence of these substrates. With mycelium $46 \mathrm{hr}$. old both lard oil and oleic acid were oxidized rapidly. With lard oil as substrate, respiration was increased over $100 \%$ and with oleic acid the increase was as much as $200 \%$ despite the relatively high endogenous rate. The maximum rate of oxidation of lard oil and oleic acid occurred with the mycelium c. $48 \mathrm{hr}$. old; mycelium taken at $74 \mathrm{hr}$. showed lower rates of oxidation.

These changes in the ability to oxidize lard oil and oleic acid might indicate the absence of the necessary enzymes in the young mycelium or might be due to unfavourable conditions for enzyme activity in the early stages of the fermentation. In the experiment shown in Fig. 3 all the samples of mycelium were suspended in the salts solution adjusted to the $\mathrm{pH}$ of the fermentation at that time. During the fermentation the $\mathrm{pH}$ rose from an initial value of c. 5.6 to 7.5-8.0. Thus the changes in response to lard oil and oleic acid during the fermentation might be due in part to $\mathrm{pH}$. 


\section{Effect of $\mathrm{pH}$ value of suspension medium}

Preliminary experiments on the effect of $\mathrm{pH}$ on oleic acid oxidation were carried out using mycelium washed and suspended in $0.01 \mathrm{M}$-phosphate buffers and $1.0 \%(v / v)$ substrate. Although the buffers were too weak to maintain a constant pH value during the period of the experiment it was evident that the oxidation of oleic acid was markedly affected by the $\mathrm{pH}$ value, with an optimum in the region of $\mathrm{pH} 7 \cdot 0$. Endogenous respiration was unaffected by $\mathrm{pH}$ value over the range $4 \cdot 3-8 \cdot 3$. The results shown in Fig. 4

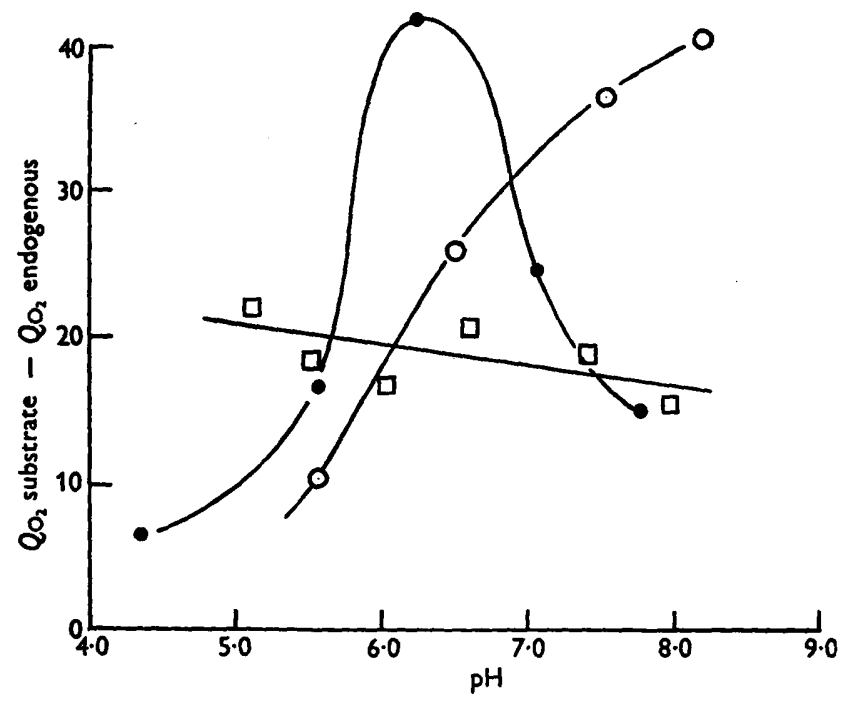

Fig. 4. Effect of pH on oxidation of lard oil, oleic acid and glucose by $P$. chrysogenum grown in a highly aerated fermentation. Glucose, $1 \%(\mathrm{w} / \mathrm{v})$, mycelium $26 \mathrm{hr}$. old, washed and suspended in $0.05 \mathrm{M}$ phosphate buffers, $\square-\square$; lard oil, $1.0 \%(\mathrm{v} / \mathrm{v})$, mycelium $44 \mathrm{hr}$. old washed and suspended in $0.05 \mathrm{M}$ phosphate buffers, $O-O$; oleic acid, $0.05 \%(\mathrm{v} / \mathrm{v})$, mycelium $51 \mathrm{hr}$. old washed and suspended in $0.1 \mathrm{M}$-phosphate buffers,

on the effect of $\mathrm{pH}$ value on oleic acid oxidation were obtained using $0 \cdot 1 \mathrm{M}$ phosphate buffers and $0.05 \%(\mathrm{v} / \mathrm{v})$ substrate. Under these conditions the $\mathbf{p H}$ remained constant during the experiment, and the relatively high concentration of phosphate did not appear to exert any unfavourable effect on respiration, although the optimum $\mathrm{pH}$ value under these conditions $(c . \mathrm{pH} 6 \cdot 4)$ was somewhat lower than indicated from the experiments with $0.01 \mathrm{M}$ buffers. Although there was a sharp optimum in the region of $\mathrm{pH} 6.4$ there was measurable activity as low as $\mathrm{pH} 4 \cdot 3$. At pH 5.6 (pH value of the fermentation at $21 \mathrm{hr}$.) the rate of oxygen uptake was over $100 \%$ higher than the endogenous rate.

The experiments on $\mathrm{pH}$ and lard oil oxidation were carried out using $0.05 \mathrm{M}$-phosphate buffers and $1.0 \%(\mathrm{v} / \mathrm{v})$ substrate. The effect of $\mathrm{pH}$ was again marked. The optimum $\mathrm{pH}$ appeared to be $>8 \cdot 1$, although at $\mathrm{pH} 5 \cdot 6$ oxidation of the lard oil was clearly evident, the oxygen uptake being $c .50 \%$ higher than the endogenous rate. 
Oxidation of lard oil and oleic acid was only apparent with older mycelium. Young mycelium (c. $20 \mathrm{hr}$.) showed no increase in oxygen uptake in the presence of oleic acid or lard oil as substrate when suspended at the $\mathrm{pH}$ normally prevailing at that time $(c .5 \cdot 6)$ nor when suspended at $\mathrm{pH}$ values near optimum for oxidation of these substrates by older mycelium. Oleic acid was added to suspensions of young mycelium adjusted to $\mathrm{pH} \mathrm{6.4}$ and lard oil added to suspensions adjusted to $\mathrm{pH} 7 \cdot 8$. Both substrates were present

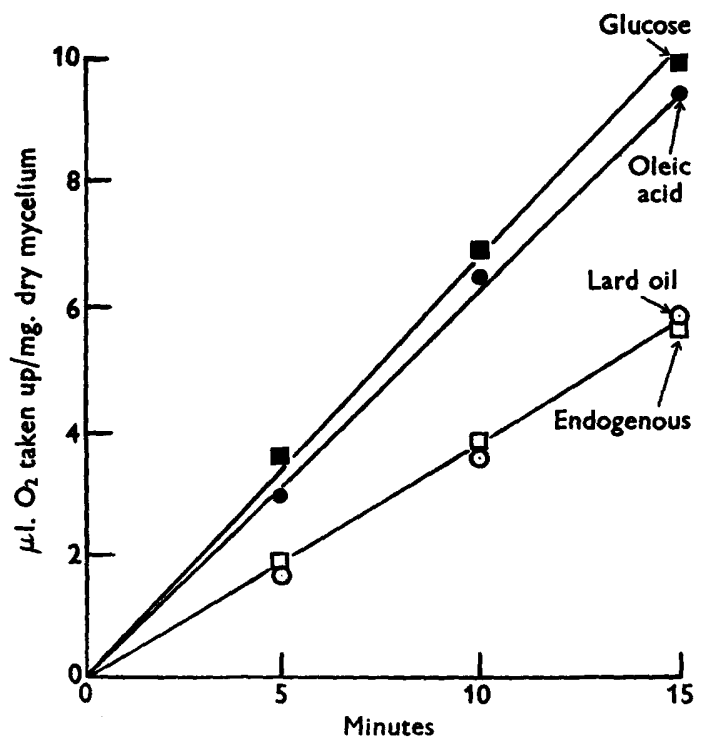

Fig. 5. Oxidation of lard oil, oleic acid and glucose by washed mycelium of $P$. chrysogenum from a shaken flask fermentation. Glucose, $1 \%(w / v)$, - ; oleic acid, $1 \%(v / v)$, - ; lard oil, $1 \%$ (v/v), $\bigcirc-0$. Endogenous respiration, $\square-\square$.

at a concentration of $1.0 \%(\mathrm{v} / \mathrm{v})$. No increase in oxygen uptake occurred over the endogenous rate, whereas at these $\mathrm{pH}$ values older mycelium oxidized oleic acid and lard oil rapidly.

In contrast to the results with lard oil and oleic acid, the oxidation of glucose was little affected by $\mathrm{pH}$ over the range 5·1-7.95 (Fig. 4).

\section{Effect of aeration during growth}

In a previous paper (Rolinson \& Lumb, 1953) it was shown that mycelium $69 \mathrm{hr}$. old, grown in a shaken flask (conditions of relatively low aeration), showed no apparent oxidation of lard oil added as sole substrate to suspensions of washed mycelium. Mycelium of similar age grown under conditions of high aeration however was able to oxidize lard oil rapidly (Fig. 3). Further experiments have shown that although the complete fat does not increase the oxygen uptake of mycelium from a shaken flask fermentation, a product of hydrolysis of lard oil such as oleic acid is oxidized rapidly (Fig. 5). In this experiment the mycelium which was $45 \mathrm{hr}$. old was washed and suspended in 
salts solution pH 6.9 and lard oil, oleic acid and glucose added as sole substrates. It will be seen that although the presence of lard oil did not increase the rate of respiration above the endogenous rate (despite the favourable $\mathrm{pH}$ for oxidation of lard oil), oxygen uptake with added oleic acid was almost as high as with glucose as substrate.

The ability of mycelium from shaken flask fermentations to oxidize oleic acid shows the same variation with age as mycelium from highly aerated fermentations. The results given in Table 2 show that whereas young mycelium showed no increase in oxygen uptake in the presence of oleic acid, with older mycelium the acid was oxidized rapidly. In this experiment the $\mathrm{pH}$ of both the 21 and the $69 \mathrm{hr}$. mycelial suspensions was adjusted to 6.5 , which is near the optimum for oleic acid oxidation as judged from Fig. 4, yet with the young mycelium respiration with oleic acid as substrate was in fact lower than the endogenous rate.

Table 2. Oxidation of lard oil and oleic acid by mycelium from a shaken flask fermentation

Mycelium washed and suspended in salts solution pH 6.5.

$\begin{array}{cccc}\begin{array}{c}\text { Age of } \\ \text { mycelium }\end{array} & \begin{array}{c}Q_{\mathrm{O}_{2}} \\ \text { endogenous }\end{array} & \begin{array}{c}1 \%(\mathrm{v} / \mathrm{v}) \\ \text { lard oil }\end{array} & \begin{array}{c}\mathrm{Q} \%(\mathrm{v} / \mathrm{v}) \\ \text { oleic acid }\end{array} \\ 21 \mathrm{hr} . & 12 \cdot 7 & 18 \cdot 8 & \mathbf{Q} \\ 69 \mathrm{hr} . & 22 & 20 & \mathbf{2 \cdot 7 5}\end{array}$

Lard oil was not normally added to shaken flask fermentations as it was to fermentations provided with high aeration because frothing did not necessitate the use of antifoam. However, when $0.25 \%$ lard oil was included in the fermentation medium the mycelium showed no significant increase in ability to oxidize lard oil.

\section{DISCUSSION}

In the highly aerated fermentations the increase in ability to oxidize lard oil during the period $20-48 \mathrm{hr}$. corresponds with the change in the respiratory quotient (R.Q.) of such mycelium reported earlier (Rolinson, 1952). At $24 \mathrm{hr}$. the R.Q. was $c$. 1.0, but at $46 \mathrm{hr}$. it had fallen to $c .0 .8$ and then continued to fall slowly as the fermentation proceeded. Also, during the first $c .30 \mathrm{hr}$. of these fermentations, lactose disappeared rapidly but, after this time, this rate was only very slow (Rolinson \& Lumb, 1953). The results reported here correspond with these previous results in that during the period when lactose disappearance was rapid, lard oil was oxidized only slowly or not at all, whereas at $48 \mathrm{hr}$. and after, when lactose was disappearing slowly, lard oil was oxidized rapidly.

In contrast with the changes in R.Q. during a highly aerated fermentation, the R.Q. of mycelium from shaken flask fermentations remained at c. 1.0 throughout the fermentation and, in the experiments described here, oxidation of lard oil by such mycelium was relatively slow during the whole fermentation. 
The absence of any increase in oxygen uptake of young mycelium when lard oil was added as substrate may not indicate the complete absence of oxidation of this substrate. The endogenous respiration in these experiments was relatively high (typical of fungi). A substrate added to a suspension of washed mycelium might well be oxidized, but at the expense of substances otherwise contributing to the endogenous respiration, and thus result in no increase in overall rate of respiration. The apparent absence of lard-oil oxidation by young mycelium may only indicate a relatively lower rate of oxidation than occurs with the older mycelium.

The change in ability to oxidize lard oil and oleic acid may be due to the synthesis of the necessary enzymes during growth. Variations in enzyme activity with age have been reported for a number of bacterial enzymes (Gale, 1943) which show little or no activity in young cells but are formed during growth and reach a maximum at the time of cessation of cell division. In the experiments described here with $\boldsymbol{P}$. chrysogenum the period when the ability to oxidize lard oil increased also corresponded with the period of maximum rate of growth. However, such a variation in enzyme activity might not be due to variation in enzyme content but to changes in the conditions for enzyme action such as changes in $\mathrm{pH}$ value of the medium or permeability of the cells during the fermentation. During the initial $24 \mathrm{hr}$. the $\mathrm{pH}$ value of the fermentation is not optimum for oxidation of either lard oil or oleic acid, but the results shown in Fig. 4 suggest that the $\mathrm{pH}$ alone would not preclude enzyme activity being apparent at this stage of the fermentation, particularly with oleic acid as substrate. However, experiments with washed mycelium suspended in buffers do not rule out the possibility that the internal $\mathrm{pH}$ of the mycelium is not the same as that of the surrounding medium. The presence during the early stages of growth of chemical factors which might inhibit enzyme action also cannot be overlooked, although this might be expected to be minimized by the washing procedure.

Variation in permeability of the mycelium to lard oil and oleic acid might also be responsible for variations in enzyme activity with age. However, experiments showed that no appreciable period of time after adding substrate (lard oil) was required before an increased rate of oxygen uptake was observed with $50 \mathrm{hr}$. mycelium, yet with young mycelium no increase in respiration over the endogenous rate was found over $30 \mathrm{~min}$. after lard oil was added. It is also not unlikely that the whole fat does not penetrate the cell but is hydrolysed outside the cell membrane. Furthermore, if the variations in enzyme activity with age were due to changes in permeability it would also be necessary to postulate a decrease in permeability after $c .48 \mathrm{hr}$. when enzyme activity declined (Fig. 3).

The inability of young mycelium to oxidize lard oil is not due only to an inability to oxidize the constituent fatty acids at this stage. The fat is apparently not hydrolysed, because glycerol, which would be liberated by hydrolysis, was readily oxidized by young mycelium at pH 5.3 (Table 1).

Oxidation of oleic acid by mycelium grown under conditions of relatively low aeration showed a similar variation with age as seen with the mycelium 
from highly aerated fermentations. The rate of oxidation of lard oil, however, was relatively low throughout the fermentation. The level of aeration during growth thus affected the ability of the mycelium to metabolize the complete fat rather than a constituent fatty acid. Although conclusions cannot be made with certainty, it seems probable that enzymes involved in the oxidation of lard oil and oleic acid are present only in low concentration in young mycelium and are formed as further growth takes place. If this is true, the degree of aeration would appear to influence the synthesis of those enzymes involved in the hydrolysis of the fat.

The authors are indebted to the late Sir Jack Drummond, F.R.S., and to Mr C. E. Coulthard for their interest in the work, to Mr R. Fawcett for assistance in carrying out the fermentations, and to Mr C. K. Mercer and Mr J. Ransom for technical assistance.

\section{REFERENCES}

Gale, E. F. (1943). Factors influencing the enzymic activities of bacteria. Bact. Rev. 7, 139.

LUMB, M. \& FAwCETT, R. (1951). Improvements in experimental fermenters. J. appl. Chem. 1, S 94.

Rourson, G. N. (1952). Respiration of Penicillium chrysogenum in penicillin fermentations. J. gen. Microbiol. 6, 336.

Roursson, G. N. \& Lumb, M. (1953). The effect of aeration on the utilization of respiratory substrates by Penicillium chrysogenum in submerged culture. J. gen. Microbiol. 8, 265.

Umbreit, W. W., BURRts, R. H. \& STAUFFer, J. F. (1945). Manometric Techniques and related Methods for the Study of Tissue Metabolism. Minneapolis, Minn.: Burgess.

(Received 7 May 1953) 